\title{
Constituintes químicos e avaliação preliminar in vivo da atividade antimalárica de Ouratea nitida Aubl (Ochnaceae)
}

\author{
Charles S. Estevam ${ }^{1}$, Fernando M. Oliveira ${ }^{1}$, Lúcia M. Conserva ${ }^{1 *}$, Lúcia de Fátima C.O. \\ Lima $^{2}$, Elaine C.P. Barros ${ }^{2}$, Angela C.P. Barros ${ }^{2}$ Eliana M.M. Rocha ${ }^{2}$ Eloísa H.A. Andrade
}

${ }^{1}$ Departamento de Química, Universidade Federal de Alagoas, 57072-970, Maceió, AL, Brasil, ${ }^{2}$ Departamento de Patologia,Universidade Federal de Alagoas, 57072-970, Maceió, AL, Brasil, ${ }^{3}$ Departamento de Botânica, Museu Paraense Emílio Goeldi, 66040-170, Belém, PA, Brasil

\begin{abstract}
RESUMO: O presente trabalho descreve o isolamento de triterpenos (ácido 3,4-seco-friedelan-3-óico, friedelina e $\beta$-amirina) e outros compostos (éster etílico do ácido $p$-hidroxibenzóico e tetracosano), bem como a avaliação preliminar in vivo da atividade antimalárica de extratos das folhas de Ouratea nitida Aubl. Análise qualitativa através de CG-EM de uma fração apolar do extrato em hexano também foi efetuada. Ésteres metílicos e etílicos dos ácidos laúrico, mirístico, palmítico, esteárico e oléico, metílicos dos ácidos pentadecanóico, heptadecanóico, araquidônico, behênico e lignocérico e o etílico do ácido linoléico foram os componentes majoritários; enquanto que o estearato de $n$-butila, o tetracosano e a 6,10,14-trimetil-2-pentadecanona foram os minoritários. Os compostos isolados foram identificados com base na análise dos dados espectrais (IV, EM e RMN, incluindo DEPT) e estão sendo descritos pela primeira vez nesta espécie.
\end{abstract}

Unitermos: Ouratea nitida, Ochnaceae, triterpenos, atividade antimalárica, CG-EM.

\begin{abstract}
Chemical constituents and in vivo preliminary evaluation of antimalarial activity of Ouratea nitida Aubl. (Ochnaceae)". This work describes the isolation of triterpenes (3,4-seco-friedelan-3-oic acid, friedelin, and $\beta$-amyrin) and other compounds ( $p$-hydroxybenzoic acid ethyl ester and tetracosane) as well as a preliminary evaluation of in vivo antimalarial activity of the extracts from the leaves of Ouratea nitida Aubl. Qualitative analysis by GC-MS of an apolar fraction from the hexane extract was also carried out. Methyl and ethyl esters of lauric, myristic, palmitic, stearic and oleic acids, methyl ester of pentadecanoic, heptadecanoic, arachidonic, beenic and lignoceric acids, and ethyl ester of linoleic acid were found to be the main constituents while $n$-butyl stearate, tetracosane and 6,10,14-trimetthyl-2-pentadecanone were the minor. All isolated compounds were identified on basis of the spectral data (IR, MS and NMR, including DEPT) and are being described for the first time in this specie.
\end{abstract}

Keywords: Ouratea nitida, Ochnaceae, triterpenes, antimalarial activity, GC-MS.

\section{INTRODUÇÃO}

O gênero Ouratea consiste de cerca de 300 espécies distribuídas principalmente nos trópicos da América do Sul (De Carvalho et al., 2000). São utilizadas na medicina popular como adstringentes, tônicas, estomáquicas, vermífugas (Braga, 1960), em distúrbios gástricos e reumatismo (Mbing et al., 2003a). Estudos químicos realizados em espécies deste gênero revelou ocorrência de flavonóides (Delle Monache et al., 1967; Moreira et al., 1994; 1999; Velandia et al., 1998a;), norisoprenóides (Velandia et al., 1998a), diterpenos (Velandia et al., 1998a; Felício et al., 2004), triterpenos (Mbing et al., 2003b), lignanas (Velandia et al., 1998a), biflavonóides (Felício et al., 1995; 2001; 2004; Velandia et al., 1998b; Mbing et al., 2003b), além de ácidos e ésteres alifáticos identificados nas raízes de $O$. hexasperma (St. Hill) Baill.
(Moreira et al., 1994) e nos frutos de O. parviflora Baill. (Paulo et al., 1986).

A espécie Ouratea nitida Aubl. é uma árvore amplamente distribuída em áreas litorâneas do nordeste brasileiro. Nenhum estudo químico ou biológico foi relatado com esta espécie.

\section{MATERIAL E MÉTODOS}

Métodos gerais

As cromatografias em coluna foram efetuadas em gel de sílica (70-230 e 230-400 mesh, Merck) e em Sephadex LH-20 (Pharmacia). Os espectros IV foram registrados em pastilhas de $\mathrm{KBr}$ utilizando um espectrofotômetro Perkin-Elmer, FT-IR 1750. Os espectros de RMN $\left({ }^{1} \mathrm{H}\right.$ : 200 e $500 \mathrm{MHz} ;{ }^{13} \mathrm{C}$ : 50 e 125 
$\mathrm{MHz}$ ) foram obtidos em espectrômetros Mercury-Varian 200 e Brucker DRX-500, respectivamente. O sinal residual do solvente ou o TMS foram utilizados como referência interna. A análise em CG-EM foi efetuada usando uma combinação de CG-FID (HP, modelo 5890), coluna capilar de CP-Sil-8CB (25 m x 0,25 mm d.i., 0,25 $\mu \mathrm{m}$ de espessura do filme) e CG-EM (Varian 3200 e Finnigan Incos-XL) em uma coluna de sílica fundida DB-5 (30m x $0,25 \mathrm{~mm}$ d.i., $0,25 \mu \mathrm{m}$ de espessura do filme).

\section{Coleta e identificação do material vegetal}

As folhas de um espécime de Ouratea nitida Aubl. foram coletadas, em agosto de 1998, na Área de Proteção Ambiental de Santa Rita (Mucuri), município de Marechal Deodoro, Alagoas, Brasil. A espécie foi identificada pela botânica Rosangela Pereira de Lyra Lemos do Instituto do Meio Ambiente do Estado de Alagoas (IMA/AL), onde um exemplar foi depositado (MAC-9934).

\section{Extração e isolamento dos constituintes químicos}

As folhas secas e pulverizadas (1170 g) foram extraídas através de maceração com EtOH 90\%. Após remoção do solvente em evaporador rotatório, o extrato (320 g) foi suspenso em solução $\mathrm{MeOH}-\mathrm{H}_{2} \mathrm{O}$ (3:2) e extraído sucessivamente com $\mathrm{C}_{6} \mathrm{H}_{14}, \mathrm{CHCl}_{3}$ e AcOEt. $\mathrm{O}$ extrato em $\mathrm{C}_{6} \mathrm{H}_{14}$ (31,58 g), parcialmente ativo nos ensaios antimaláricos in vivo, foi fracionado em coluna de gel de sílica (70-230 mesh) com misturas de hexano e AcOEt em proporções crescentes de polaridade. As frações recolhidas foram monitoradas em camada delgada e reunidas. O material oleoso e apolar, obtido em $\mathrm{C}_{6} \mathrm{H}_{14}$, das frações reunidas 15-49 (1,63 g) foi suspenso em solução aquosa de $\mathrm{NaOH} 4 \%$ e extraído com $\mathrm{C}_{6} \mathrm{H}_{14}$. A porção neutra solúvel em hexano (1,27 g) foi submetida à análise qualitativa através de CG-EM. As frações remanescentes, após sucessivos fracionamentos cromatográficos em coluna de gel de sílica, permeação em gel e cristalizações com $\mathrm{MeOH}$ forneceram três triterpenos [ácido 3,4-seco-friedelan-3-óico (1, 0,052 g), friedelina $(2,1,88 \mathrm{~g})$ e $\beta$-amirina $(\mathbf{3}, 0,418 \mathrm{~g})$ ] e um alcano [tetracosano (4, 0,661 g)]. Do extrato em $\mathrm{CHCl}_{3}(42,1 \mathrm{~g})$ foram isolados a friedelina (2, 3,82 g) e o éster etílico do ácido p-hidroxibenzóico (5, 0,078 g), sendo este último possivelmente um artefato formado durante o processo de extração.

Ácido 3,4-seco-friedelan-3-óico (1). Sólido amorfo; IV (KBr) cm ${ }^{-1}$ : 3000-2500, 2928, 2872, 1707, 1467, 1390, 943; RMN ${ }^{1} \mathrm{H}\left(500 \mathrm{MHz}, \mathrm{CDCl}_{3}\right): \delta 0,81$ $(3 \mathrm{H}, \mathrm{t}, J=6,9 \mathrm{~Hz}), 2,40-2,32(2 \mathrm{H}, \mathrm{m}), 0,76,0,92,0,96$, 0,97, 0,99, 1,18, 1,27 (3H, s cada); RMN ${ }^{13} \mathrm{C}(125 \mathrm{MHz}$, $\mathrm{CDCl}_{3}$ ): $\delta$ 21,03 (C-1), 38,28 (C-2), 179,41 (C-3), 37,80 (C-4), 39,56 (C-5), 39,01 (C-6), 18,08 (C-7), 52,55 (C-8), 37,46 (C-9), 59,37 (C-10), 35,28 (C-11), 29,97 (C-12),
38,90 (C-13), 39,26 (C-14), 32,24 (C-15), 36,03 (C-16), 30,18 (C-17), 42,79 (C-18), 35,14 (C-19), 28,14 (C-20), 32,81 (C-21), 39,01 (C-22), 7,40 (C-23), 19,33 (C-24), 17,92 (C-25), 20,13 (C-26), 18,74 (C-27), 32,09 (C-28), 34,96 (C-29), 31,82 (C-30); EM m/z: 444 (M, 6\%), 429 (12), 415 (2), 319 (18), 290 (23), 256 (1), 218 (100).

* O padrão de hidrogenação de cada carbono foi deduzido pelo experimento DEPT.

\section{Análise dos constituintes através de CG-EM}

A análise qualitativa dos constituintes foi efetuada utilizando-se uma combinação de CG-FID e CG-EM. Em CG, os componentes foram analisados usando uma coluna capilar de sílica fundida CP-Sil-8CB (25 m x 0,25 mm d.i., 0,25 $\mu \mathrm{m}$ de espessura do filme). O cromatógrafo foi equipado com um detector em chama, conectado com um integrador eletrônico da série II HP 3396. Nas análises em CG-EM utilizou-se um sistema Finnigan Mat INCOS-XL, equipado com uma coluna de sílica fundida DB-5 (30 m x 0,25 mm, 0,25 $\mu \mathrm{m}$ de espessura do filme), conforme as condições experimentais descritas por Palmeira Júnior e colaboradores (2004).

A percentagem da composição da porção volátil foi computada pela área dos picos nos respectivos cromatogramas e os componentes individuais foram identificados pela comparação dos espectros de massas e dos índices de retenção com os de compostos padrões previamente analisados e armazenados em banco de dados ou pela comparação com espectros de massas relatados na literatura (Jennings \& Shibamoto, 1980; Adams, 1989; 1995). Os índices de retenção foram calculados usando a série homóloga de $n$-alcanos em coluna DB-5.

\section{Avaliação da atividade antimalárica}

A avaliação preliminar in vivo da atividade antimalárica foi efetuada utilizando-se camundongos albinos (Mus musculus), pesando cerca de $16-20 \mathrm{~g}$ cada, criados no Biotério Setorial do Centro de Ciências Biológicas da Universidade Federal de Alagoas. O ensaio foi realizado através do teste supressivo descrito por Peters (1980), no qual os animais foram infectados, por via intraperitoneal, com cepa NK-65 do Plasmodium berghei $\left(10^{6}\right.$ hemácias parasitadas/animal). Os extratos das folhas $\left(\mathrm{C}_{6} \mathrm{H}_{14}, \mathrm{CHCl}_{3}\right.$, AcOEt e $\left.\mathrm{MeOH}-\mathrm{H}_{2} \mathrm{O}\right)$, nas doses de 250, 500 e $1000 \mathrm{mg} / \mathrm{Kg}$, foram administrados por via oral durante quatro dias consecutivos e a atividade antimalárica foi determinada pela percentagem da redução da parasitemia dos grupos tratados quando comparados com o grupo controle (não tratados). A supressão da parasitemia entre os grupos foi analisada pelo teste $t$ de Student e considerado estatisticamente significativo para $\mathrm{p}<0,05$. Difosfato de cloroquina (50 mg/kg) foi usado como controle positivo. 


\section{RESULTADOS E DISCUSSÃO}

Dentre os extratos submetidos a ensaios antimaláricos in vivo, somente o hexano apresentou resultados promissores nas doses testadas (250, 500 e $1000 \mathrm{mg} / \mathrm{kg}$ ), uma vez que a redução da parasitemia induzida pelo $P$. berghei foi de 47,87, 77,95 e 51,04\%, respectivamente. Estes dados foram considerados significativos para $\mathrm{p}<0,05$ e a inibição do crescimento do parasito pela cloroquina (controle positivo) foi de $100 \%$. Este extrato foi fracionado em coluna de gel de sílica e forneceu as substâncias 1-4.

O espectro IV da substância 1 revelou bandas de absorção em 3000-2500, 1707 e 943 cm-1 condizente com a presença de um grupo carboxílico e o espectro de RMN ${ }^{1} \mathrm{H}$, obtido a $500 \mathrm{MHz}$ em $\mathrm{CDCl}_{3}$, evidenciou a presença de um sinal triplo em $\delta 0,81(3 \mathrm{H}, \mathrm{t}, J=6,9 \mathrm{~Hz})$ sugerindo a presença de um grupo etila. $\mathrm{O}$ espectro de $\mathrm{RMN}{ }^{13} \mathrm{C}$ associado ao DEPT $135^{\circ}$ permitiu o reconhecimento de 30 átomos de carbono (7 não hidrogenados, 3 monoidrogenados, 12 diidrogenados e 8 triidrogenados). Dentre os quais ressalta-se absorções para um grupo carboxílico $(\delta 179,41)$ e um grupo metílico altamente protegido $(\delta 7,40)$. Estas observações, associada à presença no espectro de massas do pico do íon molecular em $m / z 444$ (6) e comparação com dados descritos na literatura (David et al., 2003) permitiram propor a estrutura de um triterpeno pentacíclico do tipo 3,4-secofriedelano, o ácido 3,4-seco-friedelan-3-óico. Este ácido foi primeiramente obtido como um dos produtos da decomposição fotoquímica da friedelina (Aoyagi et al., 1973). Entretanto, a análise comparativa das condições experimentais descritas na literatura (Aoyagi et al., 1973) para obtenção de derivados 3,4-seco com as do presente trabalho permitiu deduzir que o triterpeno $\mathbf{1}$ isolado é um produto natural e não um produto da degradação de 2. Além disso, esta substância foi isolada anteriormente somente das partes aéreas de Stigmaphyllum paralias A. Juss. (Malpighiaceae) como um produto natural (David et al., 2003).

As estruturas dos triterpenos [friedelina (2) e $\beta$-amirina (3)] foram identificadas pela comparação dos dados espectrais obtidos com os da literatura (David et al., 2003; Mahato \& Kundu, 1994).

A substância $\mathbf{5}$ foi identificada com base na análise conjunta dos dados de RMN. O espectro de RMN ${ }^{1} \mathrm{H}$, obtido a $200 \mathrm{MHz}$, revelou sinais condizentes com a presença de um anel aromático do tipo 1,4-dissubstituído [ $\delta 6,88$ e $\delta 7,92$ (d, $J=8,6 \mathrm{~Hz}$, cada)] e de um grupo etoxila [ $\delta 1,35(\mathrm{t}, J=7,2 \mathrm{~Hz})$ e $\delta 4,33(\mathrm{q}, J=7,3 \mathrm{~Hz})]$. A análise do espectro RMN ${ }^{13} \mathrm{C}$-APT, obtido a $50 \mathrm{MHz}$, permitiu reconhecer sinais para um grupo éster $(\delta 167,23)$, grupo etoxila [ $\delta 60,98\left(\mathrm{CH}_{2}\right)$ e $\left.\delta 14,25\left(\mathrm{CH}_{3}\right)\right]$ e para um anel aromático 1,4-dissubstituído [ $\delta$ 115,27 $(\mathrm{CH}), \delta$ 131,87 (CH), $\delta 122,20$ (C) e $\delta 160,59(\mathrm{C})]$.

A análise qualitativa através de CG-EM da porção neutra mostrou que os componentes voláteis predominantes foram ésteres metílicos e etílicos dos<smiles>CCC1(C)CCC2(C)C(CC[C@]3(C)C4CC(C)(C)CCC4(C)CCC23C)[C@]1(C)CCC(=O)O</smiles>

1<smiles>CC1(C)CCC2(C)CC[C@]3(C)C(=CCC4[C@@]5(C)CCC(O)C(C)(C)C5CC[C@]43C)C2C1</smiles>

3<smiles>C[C@H]1C(=O)CC[C@H]2[C@@H]3CC[C@]4(C)C5CC(C)(C)CCC5(C)CC[C@]4(C)[C@]3(C)CC[C@@]21C</smiles>

2<smiles>CCOC(=O)c1ccc(O)cc1</smiles>

5 
ácidos laúrico, mirístico, palmítico, esteárico e oléico; metílicos dos ácidos pentadecanóico, heptadecanóico, araquidônico, behênico e lignocérico, bem como o éster etílico do ácido linoléico. Por sua vez, os componentes minoritários foram o estearato de $n$-butila, o tetracosano e a 6,10,14-trimetil-2-pentadecanona.

\section{AGRADECIMENTOS}

Os autores expressam seus agradecimentos ao CNPq/PADCT II e FAPEAL pelo auxílio financeiro, ao Laboratório de Tecnologia Farmacêutica da Universidade Federal da Paraíba e ao CENAREMN da Universidade Federal do Ceará, pelos espectros de RMN.

\section{REFERÊNCIAS}

Adams RP 1989. Identification of essential oils by ion trap mass spectroscopy. New York: Academic Press.

Adams RP 1995. Identification of essential oil components by gas chromatography/mass spectroscopy. Illinois: Allured Publishing Corporation.

Aoyagi R, Tsuyuki T, Takai M, Takahashi T 1973. Mechanism of formation of unusual photodegradation products from friedelin. Tetrahedron 29: 4331-4340.

Braga R 1960. Plantas do Nordeste, especialmente do Ceará. Fortaleza: Imprensa Oficial.

David JM, Santos FA, Guedes MLS, David JP 2003. Flavonóide e triterpenos de Stigmaphyllum paralias. Quím Nova 26: 484-487.

De Carvalho MG, de Carvalho GJA, Braz Filho R 2000. Chemical constituents from Ouratea floribunda: complete ${ }^{1} \mathrm{H}$ and ${ }^{13} \mathrm{C}$ NMR assignments of atranorin and its new acetyl derivative. $J$ Braz Chem Soc 11: 143-147.

Delle Monache F, D'Albuquerque IL, Ferrari F, MariniBettólo GB 1967. A new catechin and a dimeric proanthocyanidin from Ouratea sp. Tetrahedron Lett 8: 4211-4214.

Felício JD, Gonçalez E, Braggio MM, Costantino L, Albasini A, Lins AP 1995. Inhibition of lens aldose reductase by biflavones from Ouratea spectabilis. Planta Med 61: 217-220.

Felício JD, Rossi MH, Braggio MM, Gonçalez E, Pak A, Cordeiro I, Felício RC 2004. Chemical constituents from Ouratea parviflora. Biochem Syst Ecol 32: 7981.

Felício JD, Rossi MH, Park HR, Gonçalez E, Braggio MM, David JM, Cordeiro I 2001. Biflavonoids from Ouratea multiflora. Fitoterapia 72: 453-455.

Jennings W, Shibamoto T 1980. Qualitative analysis of flavour and fragrance volatiles by glass capillary gas chromatography. New York: Academic Press.

Mahato SB, Kundu AP $1994 .{ }^{13} \mathrm{C}$ NMR spectra of pentacyclic triterpenoids - A compilation and some salient features. Phytochemistry 37: 1517-1575.

Mbing JN, Pegnyemb DE, Tih RG, Sondengam BL, Blond A, Bodo B 2003a. Two biflavonoids from Ouratea flava stem bark. Phytochemistry 63: 427-431.

Mbing JN, Bassomo MY, Pegnyemb DE, Tih RG, Sondemgam BL, Blond A, Bodo B 2003b. Constituents of Ouratea flava. Biochem Syst Ecol 31: 215-217.

Moreira IC, Sobrinho DC, de Carvalho MG, Braz-Filho R 1994. Isoflavanone dimers hexaspermone A, B and C from Ouratea hexasperma. Phytochemistry 35: 15671572.

Moreira IC, de Carvalho MG, Bastos ABFO, Braz-Filho R 1999. A flavone dimer from Ouratea hexasperma. Phytochemistry 51: 833-838.

Palmeira Júnior SF, Moura FS, Alves VL, de Oliveira FM, Bento ES, Conserva LM, Andrade EHA 2004. Neutral components from hexane extracts of Croton sellowii. Flavour Frag J 19: 69-71.

Paulo MQ, Lima EO, Maia RF, Xavier Filho L 1986. Antimicrobial activity of the oil of the fruit of Ouratea parviflora Baill (Ochnaceae). CCS 8: 19-21.

Peters W 1980. Chemotherapy of malaria. In: Kreier JP Malaria: epidemiology, chemotherapy, morphology and metabolism. New York: Academic Press, p. 145283.

Velandia JR, de Carvalho MG, Braz Filho R 1998a. Novel trichloro- and tetrachloroisoflavone isolated from Ouratea semiserrata. Nat Prod Lett 12: 191-198.

Velandia JR, de Carvalho MG, Braz Filho R 1998b. Ácido ent16a,17-diidroxicauran-19-óico isolado de Ouratea semiserrata e os desafios estereoquímicos dos carbonos quirais C-4 e C-16. Quím Nova 21: 397404. 